Jannis Androutsopoulos (Hamburg)

\title{
Digitalisierung und soziolinguistischer Wandel
}

\section{Der Fall der digitalen Interpunktion}

\begin{abstract}
Der Beitrag diskutiert Interpunktion als ein Teilsystem des Deutschen, an dem das Verhältnis zwischen Sprachwandel und dem gesamtgesellschaftlichen Wandelprozess der Digitalisierung besonders gut ablesbar ist. Der Gebrauch von Interpunktionszeichen im interaktionsorientierten digitalen Schreiben ist gekennzeichnet durch eine distributive Umstrukturierung des Interpunktionsinventars sowie durch Prozesse der Pragmatikalisierung, Syntaktisierung und Ikonizität, die verschiedene Satzzeichen betreffen. In der privaten und öffentlichen metasprachlichen Reflexion zeigen sich eine nicht-binäre Wahrnehmung digitaler Interpunktion und eine Registrierung einzelner Interpunktionspraktiken mit sozialen Gruppen. Die Verlagerung interpersonaler Kommunikation auf digitale Schriftlichkeit führt insgesamt zu einer Ausfächerung des interaktionalen Umgangs mit Interpunktion und zur Herausbildung domänenspezifischer Interpunktionsstile.
\end{abstract}

\section{Einleitung}

Unter ,digitale Interpunktion“ ist der Gebrauch von Interpunktionszeichen in der digitalen schriftbasierten Interaktion zu verstehen (Androutsopoulos 2018, i. Ersch.). Der relevante Gegenstandsbereich beschränkt sich dabei ausdrücklich auf das interaktionsorientierte Schreiben (Beißwenger/Storrer 2012), bei dem zwei oder mehrere Personen durch digital vernetzte Geräte und Software-Applikationen Beiträge austauschen und interaktionale Sequenzen aufbauen. Zwar ist die Abgrenzung zwischen text- und interaktionsorientiertem Schreiben nicht immer leicht, der Grundgedanke ist jedoch, dass nicht digitale Kommunikationsmedien als solche, sondern erst die Bedarfe der durch sie vollzogenen Interaktion eine Voraussetzung für Innovation und Wandel im Gebrauch von Interpunktionszeichen in der digitalen Schriftlichkeit darstellen.

Verglichen mit Emojis sind Interpunktionszeichen (IPZ) im digitalen Sprachgebrauch weit weniger erforscht und in der Öffentlichkeit beachtet. Zwar wird die Relevanz grafischer Variation in der linguistischen Onlineforschung bereits ab 
Mitte der 1990er Jahre diskutiert, IPZ wurden jedoch zunächst nur im Hinblick auf ihren unkonventionellen bzw. normabweichenden Gebrauch betrachtet. So z.B. wurden das Fehlen von Satzzeichen auf das Streben nach sprachlicher Ökonomie beim schnellen Chatten zurückgeführt, die Iteration von Frage- und Ausrufezeichen als Mittel von Expressivität unter Bedingungen kommunikativer Informalität bewertet (vgl. Bieswanger 2013; Androutsopoulos 2018). Spätere empirische Forschung konstatiert Genderunterschiede bei der Frequenz von Ausrufezeichen, der Auslassung von Punkt und Komma und dem Gebrauch des Apostrophs (Herring/Zelenkauskaite 2008; Squires 2012). Neuere Forschungsarbeiten wenden sich dem Gebrauch einzelner IPZ im sequenziellen Verlauf digitaler Interaktionen zu und beschreiben Interpunktion als genuin grafische Ressource der Koordination schriftbasierter Interaktion (Ong 2011; Vandergriff 2013; Busch 2017; McSweeney 2018; Androutsopoulos 2018). Auf diese Arbeiten komme ich im Folgenden zurück.

Dieser Beitrag untersucht Formen und Funktionen digitaler Interpunktion im Deutschen unter zwei Gesichtspunkten. Erstens wird gezeigt, dass in der medialen Ökologie der digitalen Kommunikation verschiedene IPZ pragmatische und strukturelle Aufgaben wahrnehmen. Zweitens wird am Beispiel der Interpunktion der oft gestellten, aber noch nicht geklärten Frage nach dem Verhältnis zwischen Sprachwandel und digitaler Kommunikation nachgegangen (Haase et al. 1997; Androutsopoulos 2011). Mit dem Konzept des soziolinguistischen Wandels wird argumentiert, dass mit der gesamtgesellschaftlichen Durchdringung der digitalen Schriftlichkeit mehrere IPZ neue Funktionen gewinnen, um Bedarfen der Kontextualisierung und Verständnissicherung in der schriftbasierten Interaktion entgegenzukommen. Daraus hervorgehende Sprachwandelprozesse werden auf drei Ebenen untersucht: (a) Gebrauchsfrequenz von IPZ im informellen digitalen Schreiben, (b) Gebrauchsmuster ausgewählter IPZ in einer Spannung zwischen syntaktischen und pragmatischen Aufgaben und (c) gesellschaftliche Wahrnehmung und Bewertung der digitalen Interpunktion.

Die herangezogenen empirischen Daten entstammen verschiedenen Quellen. Einen wichtigen Teil macht das Promotionsprojekt von Florian Busch über digitale Schreibregister aus (Busch 2020; Androutsopoulos/Busch 2020). Seine Daten umfassen u.a. 23 individuelle Textportfolios mit Samples aus schulischem und digital informellem Schreiben sowie sprachreflexive Gruppeninterviews mit 16 Proband/innen. Die Textportfolios enthalten 47 WhatsApp-Chatverläufe mit insg. 18.802 Nachrichten und 151.970 Worttokens. Weitere untersuchte Daten entstammen Facebook-Profilen von Hamburger Schüler/innen, einem Korpus von WhatsApp-Konversationen unter jungen Akademiker/innen sowie Belege für die Thematisierung bzw. Stilisierung von digitaler Interpunktion im Mediendiskurs (vgl. Androutsopoulos 2018). 
Im folgenden Abschnitt (Kap. 2) werden das Konzept des soziolinguistischen Wandels und das Verhältnis zwischen Digitalisierung und Interpunktionswandel umrissen. Daran anschließend (Kap. 3-5) werden Formen, Praktiken und Ideologien von digitaler Interpunktion exemplarisch untersucht. Abschließend (Kap. 6) werden Implikationen für die standardsprachliche Interpunktionsnorm und die Entwicklung von Schreibrepertoires im digitalen Zeitalter diskutiert.

\section{Digitalisierung, Kontextualisierung und Sprachwandel}

Schon seit mehr als 20 Jahren wird das Verhältnis zwischen internetbasierter Kommunikation und Sprachwandel vielfältig und kontrovers diskutiert. Manchmal findet dies unter kulturpessimistischen und latent technophoben Vorzeichen statt, indem ein Verlust sprachlicher Kompetenzen durch das Internet heraufbeschworen wird (vgl. Baron 2008; Dürscheid/Frick 2016). In der empirischen Linguistik pendeln die Positionen zwischen Dramatisierung und Zurückhaltung. Während Sigurd Wichter (1991, S. 89) voraussagt, dass ,die neuen Entwicklungen durchaus die Auswirkungen erreichen können, die dem Buchdruck zu Beginn der Neuzeit oder der Fernübertragungstechnik im Anfang des 20. Jahrhunderts zukommen“, ist nach David Crystal (2011, S. 57) Internetkommunikation noch so rezent, dass jenseits recht offensichtlicher Phänomene wie lexikalische Innovationen und Bildzeichen kein grundlegender Sprachwandel diagnostiziert werden könne. Analytisch werden Listen innovativer Phänomene vorgelegt und nach Strukturbereichen klassifiziert (vgl. Androutsopoulos 2011; Dürscheid/Frick 2016), wobei ihre Persistenz und Entwicklung hin zu gesamtgesellschaftlichem Sprachwandel nicht immer nachzuweisen sind. So führt die viel beachtete Arbeit von Haase et al. (1997) über „Internetkommunikation und Sprachwandel“ zahlreiche Phänomene auf grammatischer, lexikalischer und diskurspragmatischer Ebene an, wovon viele aus heutiger Sicht als Kuriositäten der frühen Internetkultur einzustufen sind (vgl. Androutsopoulos 2011).

Im Unterschied zu Haase et al. (1997) ist der hier vorgelegte Zugang zum Verhältnis von Digitalisierung und soziolinguistischem Wandel präziser und zugleich umfassender. Präziser, weil nur das Teilsystem der Interpunktion (Bredel 2011) unter die Lupe genommen wird, und umfassender, weil das hier zugrunde gelegte Konzept des soziolinguistischen Wandels (sociolinguistic change, Coupland 2009) den variationslinguistischen Fokus auf sprachstrukturellen Wandel erweitert. Mein Interesse richtet sich auf Sprachwandelprozesse in spätmodernen Gesell- 
schaften, deren sprachliche Trends und Gebrauchsstandards durch medialen Sprachgebrauch und mediale Diskurse geprägt sind (vgl. Coupland 2014; Androutsopoulos 2017). Inzwischen ist es in der Soziolinguistik weitgehend Konsens, dass die Rolle von Medien im Sprachwandel nicht mehr (wie noch in der frühen korrelativen Soziolinguistik) pauschal verworfen werden kann, sondern empirisch zu prüfen ist. ${ }^{1}$

Das Konzept des soziolinguistischen Wandels wurde von Nikolas Coupland theoretisch und epistemologisch geschärft (Coupland 2009, 2014; Androutsopoulos (Hg.) 2014, 2017). Coupland kritisiert korrelativ-variationslinguistische Zugänge zum Sprachwandel als reduktionistisch, weil sie die gesellschaftliche und ideologische Dimension von Sprachwandelprozessen nicht näher erforschen. Dem hält Coupland entgegen, dass Veränderungen in den kommunikativen Praktiken und Sprachideologien (zum Begriff vgl. Busch 2019) einer Gesellschaft als integraler Bestandteil von Sprachwandelprozessen anzusehen und in ihrem Zusammenspiel mit Strukturwandel zu untersuchen sind. Gerade weit reichende Sprachwandelprozesse - etwa Standardisierung und Destandardisierung, Kreolisierung oder Revitalisierung von Minderheitensprachen - beinhalten nicht nur sprachstrukturellen Wandel, sondern eine Umstrukturierung gesellschaftlicher Sprachrepertoires und Sprachideologien. Mit dem Konzept des soziolinguistischen Wandels wird nun versucht, eine der zentralen Fragestellungen der soziolinguistischen Sprachwandelforschung - „how change [is] embedded in social practice“ (Britain 2016, S. 143) - neu zu denken, indem die mit Strukturwandel verbundenen kommunikativen Praktiken und metasprachlichen Reflexionen gesellschaftlicher Akteur/innen in den Vordergrund gerückt werden.

Die Grundlage für den hier untersuchten soziolinguistischen Wandel in der Interpunktion ist der übergreifende Prozess der Digitalisierung, worunter aus kommunikationstheoretischer Sicht die langfristige Durchdringung aller gesellschaftlicher Handlungsfelder durch Prozesse digitaler Kommunikation zu verstehen ist (Krotz/Despotovic/Kruse (Hg.) 2017). Die Digitalisierung des Alltags hat seit Ende der 1990er Jahre zu einer Umstrukturierung des gesamtgesellschaftlichen kommunikativen Haushalts geführt und die Rolle von Schriftlichkeit nachhaltig verändert. Während sich gesellschaftliche Literalität in prä-digitalen Zeiten v.a. als eine Literalität des Lesens darstellte und alltägliches Schreiben primär auf institutionelle Kontexte fokussiert war, vollzieht sich mit der Digitalisierung des kommunikativen Alltags ein Wandel hin zur einer schreibenden Gesellschaft, in der Schriftlichkeit zu einem Medium für informelle, interpersonale Kommuni-

1 Vgl. Androutsopoulos (2017); Tagliamonte (2014) und weitere Beiträge in der Debatte über „Media and language change“ im Themenheft des Journal of Sociolinguistics 8, 2, 2014. 
kation wird (Androutsopoulos 2007; Androutsopoulos/Busch 2020; Jucker/Dürscheid 2012; Beißwenger/Storrer 2012). Durch mobile Geräte entsteht eine Kultur der ständigen Verfügbarkeit mit zahlreichen digitalen Praktiken, die v.a. auf Affordanzen mobiler digitaler Kommunikationsgeräte (Smartphones) aufbauen (vgl. Baron 2008; Jones/Chik/Hafner (Hg.) 2015). Wie aktuelle Erhebungen bei Jugendlichen zeigen, muss man sich inzwischen nicht mehr fragen, wann und wozu Menschen (insbesondere Jugendliche und junge Erwachsene) digital und vernetzt schreiben, sondern unter welchen Umständen sie es nicht tun (vgl. JIMStudie 2018; Busch 2020).

Im Zuge der durch Digitalisierung vorangetriebenen Ausbreitung schriftbasierter Interaktion entstehen und konsolidieren sich grafische Mittel der Kontextualisierung digital vermittelter Beiträge. Kontextualisierung, die Kennzeichnung intendierter Kontexte und Lesarten einer Äußerung (Gumperz 1992), ist dabei als unhintergehbarer Bedarf menschlicher Kommunikation zu verstehen. Jede verbale Interaktion wirft interpretative Grundsatzfragen auf, welche die Beteiligten fürund miteinander durch nichtreferenzielle Sprachmittel implizit beantworten (Auer 1986): Wird miteinander interagiert? Wer ist an der Interaktion beteiligt? Um welche Themen geht es? Wie stehen die Beteiligten zueinander? Usw. Bei der Interaktion auf medial grafischer Grundlage mittels vernetzter (mobiler) Geräte müssen nun interpretative Hinweise auf Fragen dieser Art durch grafisch-visuelle Mittel kontextualisiert werden. Der Einsatz von Emoticons zur Kennzeichnung von Ironie ist hier ein klassisches Beispiel, und es scheint berechtigt anzunehmen, dass das Repertoire an visuellen Kontextualisierungsmitteln weiter wächst, sofern digitale Interaktion im Zuge der mobilen Messenger-Kultur an gesellschaftlicher Verbreitung gewinnt und die für digitalsprachliches Handeln verfügbaren Zeichenrepertoires ausgebaut werden (z.B. Emojis, GIFs).

Ausschlaggebend für Interpunktionswandel im digitalen Sprachgebrauch ist im hier nur knapp skizzierten Theorierahmen also eine ,Interaktionalisierung' der Interpunktion, deren Aufgabe nicht mehr nur darin besteht, Texte syntaktisch zu strukturieren, sondern auch darin, im Sinne einer visuellen Prosodie Interpretationsrahmen zu eröffnen und aufrechtzuerhalten sowie Beziehungsarbeit zu leisten. Wohlgemerkt ist dies nicht gleichzusetzen mit einer älteren Auffassung computervermittelter Kommunikation als ,verarmt' oder ,defizitär‘ gegenüber ko-präsenter Kommunikation. Digitaler Sprachwandel besteht ja nicht in einem Verlust an Komplexität, sondern in der Umstrukturierung vorhandener Zeichenrepertoires (u.a. der Interpunktion) und Entstehung neuer Zeichenrepertoires (etwa Emojis), um neu aufkommenden kommunikativen Bedarfen zu entsprechen. Allerdings ist der Bedarf an grafischer Kontextualisierung kein Garant für den Gebrauch von Satzzeichen. Denn diese sind Teil einer grafisch-visuellen Ökologie, die auch andere Mittel der grafischen Organisation (z.B. Emojis) bereit- 
hält, zu denen IPZ in einem Verhältnis der Konkurrenz und Kookkurrenz stehen (vgl. Androutsopoulos 2018 und Kap. 4). Nicht zuletzt ist es wichtig zu fragen, welche Konsequenzen der digitale Interpunktionswandel für die normierte Zeichensetzung haben könnte. Nach derzeitigem Wissensstand gehen orthografische Interpunktionsnormen durch digitalen Interpunktionswandel nicht verloren. Vielmehr ist in der informellen digitalen Schriftlichkeit eine Ausfächerung neuer Schreibregister zu konstatieren, in denen Interpunktion anders gebraucht und interpretiert wird als in der standardsprachlichen Schriftnorm (vgl. Kap. 6).

\section{Das Interpunktionsinventar im informellen digitalen Schreiben}

Erste Beobachtungen legen nahe, dass im interaktionsorientierten Schreiben eine Umstrukturierung des IPZ-Inventars stattfindet, so dass bestimmte Satzzeichen übermäßig viel, andere kaum noch gebraucht werden (Androutsopoulos 2018). Die Auswertungen von Busch (2020) erhärten diesen Eindruck. Busch ermittelt folgende Unterschiede zum kleineren schulischen Sample (Tab. 1). ${ }^{2}$

Alle Zeichen kommen sowohl in Schultexten als auch in WhatsApp-Chats vor, bestimmte Satzzeichen werden jedoch kaum noch verwendet, v.a. das Semikolon. Die normalisierten Frequenzen zeigen, dass im schulischen Schreiben insgesamt mehr interpunktiert wird als im informellen digitalen Schreiben. Allerdings kommen das Fragezeichen, der Apostroph und die Auslassungspunkte häufiger in Chats als in Schultexten vor. Die größten Unterschiede sind beim Komma und Punkt zu verzeichnen, die fast 27 bzw. 20 Mal häufiger im schulischen Schreiben Verwendung finden. Umgekehrt sind Fragezeichen und Auslassungspunkte fünf bzw. sieben Mal häufiger in den Chat-Daten. Die nachfolgende Diskussion fokussiert auf drei Zeichen, bei denen soziolinguistische Wandelprozesse gut ablesbar sind.

2 Das IPZ-Inventar wird in drei Klassennach Bredel (2011) eingeteilt. Syntaktische Zeichen (Punkt, Komma, Semikolon, Doppelpunkt) regeln die Verarbeitung im Leseprozess, indem sie z.B. auf den Abschluss einer Informationseinheit hinweisen. Kommunikative Zeichen (Ausrufe-, Frage-, Anführungszeichen und Klammern) leiten leserseitige Rollenwechsel ein. Scan-Zeichen (Apostroph, Auslassungspunkte, Divis und Gedankenstrich) weisen auf Abweichungen vom normalen Lesefluss auf Satz- oder Textebene hin. 
Tab. 1: Interpunktionszeichen auf 1.000 Wörter im Vergleich (absolute Frequenzen in Klammern; Quelle: Busch i. Ersch.)

\begin{tabular}{|c|c|c|c|}
\hline & & Schultexte & Whatsapp-Chats \\
\hline \multirow[t]{4}{*}{ Syntaktische Zeichen } & $<.>$ & $72,1(1.662)$ & $3,7(568)$ \\
\hline & $<,>$ & $54,8(1.263)$ & $2(297)$ \\
\hline & $\langle;\rangle$ & $0,2(4)$ & 0,007 (1) \\
\hline & $\langle:\rangle$ & $6,7(155)$ & 1,3 (193) \\
\hline \multirow[t]{5}{*}{ Kommunikative Zeichen } & $\langle!\rangle$ & $2,1(49)$ & $1,2(188)$ \\
\hline & $\langle ?\rangle$ & $3,3(77)$ & $17,2(2.621)$ \\
\hline & $\langle “\rangle$ & $13,6(313)$ & $0,3(47)$ \\
\hline & $<(>$ & $9,7(223)$ & $0,5(74)$ \\
\hline & \langle\rangle$\rangle$ & $11,3(261)$ & $0,5(79)$ \\
\hline \multirow[t]{3}{*}{ Scan-Zeichen } & $\langle->$ & $5,1(121)$ & $0,2(32)$ \\
\hline & $\langle>\rangle$ & $0,1(3)$ & $0,4(63)$ \\
\hline & $\langle\ldots\rangle$ & $0,9(21)$ & $6,3(952)$ \\
\hline$\Sigma$ & & $181,2(4.152)$ & $33,7(5.115)$ \\
\hline
\end{tabular}

\section{Interpunktionspraktiken im digitalen Wandel}

\subsection{Pragmatisierung: Der beitragsfinale Punkt}

Die Auslassung des Punktes am Ende digitaler Nachrichten wird gewöhnlich als Folge der grafischen Gestaltung der Nachrichtenthreads eingestuft. Da die grafische Rahmung einer Messenger-Nachricht auch ihren Abschluss markiert, scheint der finale Punkt in seiner syntaktischen Funktion zumindest am Ende der Nachricht redundant. Baron/Ling (2011, S. 59) sprechen hier vom ,Sparsamkeitsprinzip“ (,principle of parsimony“). Vor dieser Folie richtet sich nun das Interesse auf den doch - wenn auch selten - gesetzten finalen Punkt, der sich auch bei sehr kurzen Beiträgen findet. Neuere Forschungsarbeiten zeigen, dass der Abschlusspunkt eine Reihe von pragmatischen Bedeutungen gewinnt, die hier mit dem Begriff der Pragmatisierung (bzw. Pragmatikalisierung), also des Zugewinns eines sprachlichen Zeichens an pragmatischen Funktionen (Günthner/Mutz 2004), diskutiert werden. In experimentellen Studien stellen Gunraj et al. (2016) und Houghton/Upadhyay/Klin (2018) fest, dass ein Schlusspunkt eine Messenger-Nachricht 
als unaufrichtig oder ruppig erscheinen lässt. Androutsopoulos (2018) und McSweeney (2018) beschreiben die Funktion des Schlusspunktes als Signal einer thematischen oder handlungsbezogenen Grenze. Je nach Kontext kann er als Markierung einer thematischen Abgeschlossenheit, eines Beharrens auf der eigenen Position, einer fehlenden Bereitschaft zur weiteren Verhandlung oder der Dispräferenz verstanden werden. Nach McSweeney folgt im britischen Usus auf diese Bedeutung der Abgeschlossenheit eine neuere Bedeutung des Unbehagens: „before the period was 'pissed,' it was 'final'“ (McSweeney 2018, S. 95).

In den Daten von Busch (2020) gibt es mehrere Belege dafür, dass der finale Punkt die vage pragmatische Bedeutung des Beharrens bzw. der Dispräferenz aufweist. In den nachfolgenden Beispielen markiert der Informant „Frank“ mit dem Punkt Dispräferenz im zweiten Glied eines Adjazenzpaares, um Anfragen seines Freundes „Martin“ abzulehnen. Durch die Kürze der Beiträge wirkt der Punkt umso auffallender.

Beispiel 1 (Busch 2020)

Martin: Gleich GTAV?

Frank: Ne sorry.

Beispiel 2 (Busch 2020)

Martin: Hast du am Freitag also morgen Zeit?

Frank: Nein. Leider nicht.

Martin: Hast du echt keine Zeit?

In den Daten von Uhlenberg (2016; vgl. Androutsopoulos 2018, S. 736ff.) sind Punkte eines der seltensten beitragsfinalen Zeichen und stehen in der Gebrauchsfrequenz weit nach Emojis sowie Frage- und Ausrufezeichen. Eine Informantin, die insgesamt sehr wenige finale Punkte setzt, tut dies in einer längeren Chatsequenz dennoch systematisch, um eine Terminentscheidung unter mehreren Freundinnen im Gruppenchat festzulegen. So ergeben sich Kontrastbelege wie die im Beispiel 3.

Beispiel 3 (vollständige Beiträge; Uhlenberg 2016)

(a) Also wir kommen auch um $21 \mathrm{~h}$ zur s-Bahn $(:)$

(b) Ok, dann nehmen wir jetzt den 1. Mai.

Beiträge wie (3a) sind typisch für den Duktus dieser Informantin generell, abschließende Punkte wie in (3b) tauchen mehrmals auf, wenn sie versucht, Druck auf die Gruppenchat-Mitglieder auszuüben und anzudeuten, dass sie den gesetzten Termin nicht weiter verhandeln möchte. Eben dann schlägt ihr ganzer Schreibstil ins Förmlichere um, dessen Teil auch die orthografisch korrekte Punktsetzung ist. 
Die Pragmatisierung des Schlusspunktes führt vermutlich zu einer höheren interaktionalen Salienz des Zeichens, dem Intentionalität zugeschrieben wird. Der Punkt wird im Zusammenhang mit dem propositionalen Gehalt bzw. der Sprechhandlung der Bezugsäußerung sowie ihrer sequenziellen Stellung interpretiert. Seine Bedeutung ist dabei unscharf und variabel. Mal kann sie mit „ich bin sauer“, mal mit ,ich will nicht weiter darüber reden“ umschrieben werden. In Anlehnung an das soziolinguistische Konzept des indexikalen Felds (Eckert 2008) ist festzuhalten, dass der beitragsfinale Punkt keine stabile pragmatische Bedeutung aufweist, sondern ein Feld von Bedeutungspotenzialen eröffnet, woraus im situierten Sprachgebrauch des Zeichens einzelne Bedeutungsnuancen selegiert werden.

\subsection{Multifunktionalität und Syntaktisierung: Auslassungspunkte}

Auslassungspunkte stechen in den vorliegenden Daten durch ihre hohe Frequenz und flexible Topologie hervor. Pragmatische Funktionen, die Meibauer (2007) für diverse Textsorten sowie Ong (2011) und Vandergriff (2013) für digitale Interaktionen beschreiben, finden sich auch bei Busch (2020) und Androutsopoulos (2020) wieder. In topologischer Perspektive sind vier Positionen des Zeichens in digitalen Interaktionen zu unterscheiden, die auch miteinander kombiniert auftreten:

- Beitragswertige Auslassungspunkte machen eine komplette Nachricht aus, ihre Funktion ist daher nur sequenziell zu erschließen. Je nach Kontext können sie u.a. stille Zustimmung oder Dispräferenz zum Ausdruck bringen.

- In beitragsfinaler Position ist die schulgrammatische Funktion der Auslassungspunkte als Marker für fehlendes lexikalisches oder syntaktisches Material in den digitalen Daten praktisch obsolet. Zentral im digitalen Gebrauch ist die Andeutungsfunktion (Meibauer 2007; Bredel 2011) der beitragsfinalen Auslassungspunkte, die (auch nach vollständigen Sätzen) signalisieren, dass zum relevanten Sachverhalt auch Weiteres, leicht zu Erschließendes, gesagt werden könnte.

- In der seltener belegten beitragsinitialen Position zeigt sich eine pragmatischtextuelle Leistung, die Meibauer (2007) als „Verbindungsfunktion“ beschreibt. Auslassungspunkte kennzeichnen dann einen Beitrag bzw. eine Texteinheit als Fortsetzung einer anderen lokal präsenten Texteinheit.

- Die beitragsmediale Position ist in den Daten von Busch (2020) und Androutsopoulos (2020) bei weitem am häufigsten. Hier fungieren Auslassungspunkte als segmentierende Zeichen (vgl. bereits Raclaw 2006) und treten funktionsäquivalent zu syntaktischen Satzzeichen (Punkt und Komma) auf. 
Eine ,Syntaktisierung،, d.h. ein Zugewinn syntaktischer Funktionen, kann insb. für beitragsmediale Auslassungspunkte konstatiert werden. In den Daten treten sie als häufigstes, z.T. alleiniges Zeichen der Binnengliederung eines Beitrags auf und übernehmen damit die Funktion, die üblicherweise syntaktische IPZ (Punkt und Komma) erfüllen. An zwei Beispielen aus Busch (2020, jeweils vollständige Beiträge) lassen sich verschiedene Segmentierungsmuster erläutern.

Beispiel 4 (Busch 2020)

Anne: Hi johanna ... ist das ok wenn nelly mit kommt ..[Emoji]

\section{Beispiel 5 (Busch 2020)}

Nils: moin moin... ich hab gehört dass ihr noch $n$ buch braucht.. :D fährst du auf dem weg zu lisa die isengard straße entlang? :D

Ein Muster, das Raclaw (2006) an amerikanischen und Androutsopoulos (2020) an griechischen Daten belegen, ist die Setzung von Auslassungspunkten als Trennmarker zwischen dem besetzten Vor-Vorfeld, wo eine Anrede, Lachausdrücke oder Partikeln stehen können, und dem Rest des Beitrags (Beispiele 4, 5). In längeren Beiträgen, die aus Platzgründen nicht angeführt werden können, erscheinen beitragsmediale Auslassungspunkte auch zwischen einzelnen Sätzen oder Satzfragmenten, die eigenständige Informationseinheiten bilden. Auch in (5) sieht man dies, wobei das Zeichen als Allograph mit zwei statt drei Punkten erscheint, während in (4) die zwei Punkte auch beitragsfinal erscheinen. In beiden Fällen signalisieren die Auslassungspunkte neben der Segmentierung auch, dass der fragliche Sachverhalt noch offen und „diskussionsfähig“ ist.

Beitragsmediale Auslassungspunkte grenzen also Informationseinheiten mit unterschiedlichem syntaktischem Status (Sätze, Satzfragmente, einzelne Wörter) voneinander ab, weswegen Androutsopoulos (2020) sie als „Allzweck-Segmentierer“ bezeichnet. Als Mittel der Binnensegmentierung von Messenger-Beiträgen in den Daten dienen allerdings auch Punkt und Komma sowie Emojis und Emoticons, die in der neueren Forschung ebenfalls als „Grenzsignale“, die den inneren Aufbau eines Beitrags strukturieren, beschrieben werden (Dürscheid/Siever 2017; Pappert 2017). Das Gesamtbild ist also das der Konkurrenz von grafischen Elementen aus mehreren Graphemklassen, die zur Binnengliederung längerer MessengerBeiträge herangezogen werden.

Die segmentierende Funktion der Auslassungspunkte wird dadurch ermöglicht, dass sie im Interpunktionssystem des Deutschen ein funktional wenig belastetes Zeichen sind (vgl. Bredel 2011), während ihre kanonische Aufgabe, syntaktische Unvollständigkeit am Satzende zu markieren, für digitale Interaktionen praktisch obsolet ist. Gleichzeitig belegen Auslassungspunkte einen größeren 
visuellen Raum als der Punkt und können deswegen schon rein assoziativ als eine Art ,gedehnter Punkt‘ verstanden werden. Sie bilden visuelle Trennmarken, deren Ausdehnung in die Fläche flexibel gehandhabt werden kann (Androutsopoulos 2020). Wie beim iterierten Ausrufezeichen sind also auch hier ikonische Zeichenbildungsverfahren (Auer 1989) im Gange. Innovativ bei den Auslassungspunkten ist insgesamt ihr Gebrauch zur Segmentierung von Beiträgen in einzelne Informationspakete einerseits, ihre vage interaktionale Bedeutung der Offenheit bzw. Nichtabgeschlossenheit andererseits. Dadurch stellen sie ein pragmatisches Gegenstück zum beitragsfinalen Punkt dar.

\subsection{Konventionalisierte Ikonizität: Das iterierte Ausrufezeichen}

Die Iteration von Frage- und Ausrufezeichen ist wohl das auffälligste Phänomen digitaler Interpunktion, das gemeinhin als expressives Verfahren verstanden wird. In der Forschung hat man die Iteration von Satzzeichen und Graphemen im Allgemeinen als Abbildung phonischer Qualitäten bzw. „Simulation“ von Mündlichkeit interpretiert (vgl. bereits Haase et al. 1997; Dürscheid/Frick 2017). Überzeugender finde ich jedoch eine Erklärung durch das semiotische Prinzip der Ikonizität (vgl. Androutsopoulos 2018 in Anlehnung an Auer 1989; Sandig 2006). Ikonische Zeichenbildungsverfahren stellen eine Analogie zwischen der semiotischen Beschaffenheit einer Äußerung und den darin ausgedrückten Handlungen oder Sachverhalten her. Die Iteration eines Zeichens bringt grundsätzlich eine Intensivierung zum Ausdruck, die je nach Kontext als Steigerung, Nachdruck oder Bekräftigung verstanden wird. ${ }^{3}$ Bezogen auf IPZ lässt sich eine einfache Ikonizitätsregel wie folgt ausdrücken: Je mehr Tokens des Zeichens, desto stärker die emotionale Beteiligung der Schreibenden an ihrer Äußerung. Es wird also dafür plädiert, die Iteration von grafischen Zeichen nicht einem entsprechenden phonischen Phänomen unterzuordnen, sondern sowohl phonisch als auch grafisch realisierte Iterationen als Ausprägungen eines gemeinsam zugrundeliegenden, d.h. intermodal operierenden, Zeichenbildungsverfahrens zu betrachten. Aus dieser Perspektive ist die Wiederholung von kommunikativen IPZ kein Versuch, eine bestimmte prosodische Struktur ins Schriftliche zu übertragen, sondern eine modalitätsspezifische Materialisierung von Ikonizität zum Zweck der expressiven Intensivierung.

3 Sandig (2006) zählt die Längung durch Graphemiteration neben grammatikalisierten bzw. usualisierten Dopplungen (bitte, bitte oder langsam-langsam) zu den ikonischen Verfahren. 
Die sprachreflexiven Interviews von Busch (2020) legen nahe, dass eine dreifache Iteration von Ausrufezeichen von norddeutschen Jugendlichen gegenwärtig als Normalfall wahrgenommen wird. Auch dieser Befund bestärkt die Vermutung, dass im interaktionsorientierten Schreiben eine Konventionalisierung von Graphemiterationen stattfindet (vgl. auch Darics 2013). Der Konventionalisierungsbegriff impliziert zweierlei: Erstens werden Iterationen zum unmarkierten Fall, sind also nicht mehr auffällig, sondern im Gegenteil hochgradig erwartbar, wobei erst lokales Wissen der Interaktionspartner/innen dafür ausschlaggebend ist, wie viele Iterationen ihnen eine besondere expressive Steigerung bedeuten. Zweitens entwickelt die Iteration von IPZ eine gewisse Signalisierungsinflation, so dass ihr einfaches Vorkommen für den Ausdruck einer sozial angemessenen expressiven Haltung nicht mehr ausreicht. Das nichtiterierte Zeichen kann folglich sogar als Kontextualisierung von Desinteresse wahrgenommen werden (vgl. Tannen 2013; Darics 2013; Androutsopoulos 2018).

\section{Interpunktionsideologien im digitalen Wandel: Ent-Binarisierung und Registrierung}

In den meisten Handlungsfeldern schriftlicher Kommunikation ist der Umgang mit IPZ in der Sprachreflexion binär organisiert: Entweder hält man die Rechtschreibregeln ein oder nicht, sei es durch Unkenntnis der Regeln oder Unachtsamkeit. Ein von dieser Bewertung losgelöster, expressiv-kreativer Umgang mit IPZ scheint nur wenigen Textsorten, etwa Privatbriefen oder Comics (SanchezStockhammer 2016), vorbehalten. Schon seit den 1990er Jahren wird Interpunktion als Beispiel für ,normabweichende“ geschriebene Sprache im Internet thematisiert (vgl. Brommer 2007, S. 329ff.; Baron 2008, S. 176; Squires 2010; Androutsopoulos 2011, S. 146). Auch heute noch wird digitale Interpunktion in den Medien oft als Problemfall gerahmt, indem z.B. Satzzeichen nur ex negativo, als normwidrig fehlende Zeichen, thematisiert werden.

$\mathrm{Zu}$ fragen ist, ob nicht genau an dieser Stelle ein sprachideologischer Wandel dahingehend stattfindet, dass digitale Interpunktion nicht mehr binär, sondern mit Bezug auf die schriftbasierte Interaktion und den individuellen Schreibstil der Kommunikationspartner interpretiert wird. Dieses Wegrücken von einer dichotomischen Richtig-Falsch-Wahrnehmung von Satzzeichen nenne ich „Ent-Binarisierung““4.

4 Der Terminus scheint in der sozialwissenschaftlichen Genderforschung gelegentlich gebräuchlich, ein interdisziplinärer Abgleich muss an dieser Stelle jedoch ausbleiben. 
Zumindest bei jüngeren, digital affinen Segmenten der Bevölkerung scheinen bestimmte IPZ im interaktionsorientierten Schreiben in erster Linie als kontextuell motivierte, stilistische Sektionen wahrgenommen zu werden. ${ }^{5}$ Dass diese Entwicklung nicht mit der Befürchtung gleichzusetzen ist, jüngere Schreiber/innen würden die Interpunktionsregeln nicht mehr beherrschen, wird im Fazit diskutiert.

Belege für diesen sprachideologischen Wandel finden sich in metadiskursiven Reflexionen im deutschsprachigen und internationalen Mediendiskurs:

- In einem Beitrag in den „New York Times“ wird David Crystal zum Thema Punktsetzung zitiert (Bilefsky 2016). Crystal nennt diverse Bedeutungsnuancen des Punktes im digitalen Sprachgebrauch: „the period is being deployed as a weapon to show irony, syntactic snark, insincerity, even aggression“. Ihm zufolge sei der Punkt sogar eine Art Emoticon geworden („has become an emoticon of sorts“).

- In einer Folge der US-amerikanischen Fernsehshow „Jimmy Kimmel live“ vom Jahr 2016 thematisiert der Moderator Kimmel den finalen Punkt satirisch als Mittel des „passiv-aggressiven“ Schreibens. Kimmel kontrastiert die Messenger-Nachrichten $<$ Sure! $>$ und $<$ Sure . $>$ - mit Ausrufezeichen und Punkt respektive - und versprachlicht unterhaltsam die durch den Punkt ausgelösten Inferenzen: „period instead of the exclamation point means either I thought we were going out just the two of us, or I don't care if Dave comes, or maybe because I don't like Dave“6.

- Ein deutscher Videobericht vom Dezember 2016 (Schlagzeile: „Darum sollten Sie WhatsApp-Nachrichten nie mit einem Punkt beenden“) bringt die Auslassung des Schlusspunktes mit der Gruppe der „WhatsApp-Profis“ in Verbindung, wobei im Videobericht zumeist junge Frauen bzw. weibliche Hände beim Lesen und Eintippen von Kurznachrichten gezeigt werden (vgl. Androutsopoulos 2018). Dadurch entsteht folgende implizite Zuschreibung: Ein ausgelassener Schlusspunkt sei im digitalen Austausch die neue Norm, jungen Frauen komme dabei eine Vorreiterrolle zu.

Dies sind drei Beispiele dafür, wie ein Satzzeichen im öffentlichen Diskurs nicht mehr auf seine Normkonformität bewertet, sondern auf seine Funktion als inter-

5 Bei einer weiterführenden Analyse müsste zwischen einzelnen IPZ-Klassen genauer unterschieden werden. Bereits im schulischen Schreiben ist das stilistische Potenzial einzelner IPZ durchaus unterschiedlich. Iterierte Ausrufezeichen z.B. sind auch in einem Schulaufsatz nicht im strengen Sinne ,falsch', sondern allenfalls ,unangemessen'. Florian Busch danke ich für Diskussion zu diesem Punkt.

6 https://www.youtube.com/watch?v=07TinrpKG6o (Stand: 1.10.2019). 
aktionale Ressource reflektiert wird. Der gesetzte bzw. nichtgesetzte finale Punkt wird mit interpersonalen und affektiven Haltungen einerseits, bestimmten sozialen Gruppen andererseits in Verbindung gebracht.

Die diskursive Verbindung zwischen Sprachzeichen und sozialen Stereotypen bzw. Aktivitätstypen wird in der neueren Soziolinguistik mit dem Konzept der Registrierung (enregisterment, Agha 2003; Busch 2019) diskutiert. Auch in den von Busch (2020) durchgeführten sprachreflexiven Interviews bringen die Informant/innen bestimmte Praktiken der Zeichensetzung mit sozialen Zuordnungen in Verbindung. Beispielsweise wird normkonforme Punktsetzung mit dem schulischen Schreiben und der Orientierung an Lehrkräften assoziiert, während iterierte kommunikative Zeichen als expressiv und indirekt als ,typisch weiblich' registriert sind (Busch 2020, Kap. 10). Eine soziale Registrierung dieser Art wird für die gesamtgesellschaftliche Wahrnehmung und Bewertung der digitalen Interpunktion jedoch erst dann relevant, wenn sie durch mediale Dissemination eine massenweise Rezeption erreicht, so dass Menschen in ihrer Anschlusskommunikation eine bestimmte Repräsentation von Satzzeichen (z.B. in einer Fernsehshow) als gemeinsam bekannt voraussetzen können (vgl. Agha 2003, S. 266; Androutsopoulos 2017, S. 410). In dieser Hinsicht ist sprachideologischer Wandel mit medialen Repräsentationen, in denen soziale Zuordnungen von sprachlichen Formen und Praktiken dargestellt und bewertet werden, eng verbunden.

Gegenwärtig sind solche Repräsentationen neben dem finalen Punkt auch für das iterierte Ausrufezeichen zu verzeichnen. Seine ehemalige Assoziation mit (weiblichen) Teenagern wird in den letzten Jahren durch eine Registrierung mit dem Rechtspopulismus abgelöst. Ein Beitrag im Zeit-Magazin (Kemper 2016) beschreibt das Ausrufezeichen als „Satzzeichen der ewig Unverstandenen, die das Gefühl haben, nicht ausreichend gehört zu werden - und als Konsequenz erst mal die Lautstärke hochdrehen“ "-, womit Iterationen des Zeichens gemeint sind. Als Ort seiner „inflationären“ Ausbreitung werden dort „Kommentarspalten auf Facebook oder unter Artikeln“ genannt.

Die neue soziale Registrierung des Ausrufezeichens geht also mit einer Rekontextualisierung vom privaten Schreiben zu den öffentlichen digitalen Plattformen (der Sozialen Medien) einher. Ihnen entstammen die abschließenden Beispiele:

Beispiel 6: Kommentare auf facebook.com/pegidaevofficial (2019)

HR: Sie bekommen langsam Angst, weil sie merken, das sich das Blatt wendet ! Macht weiter ,immer Weiter !!!!!

BR: Richtig ,denn im März stehen ja 3 Landtagswahlen an und daher fangen jetzt auch Politiker langsam an die Forderungen auszusprechen die von AfD und Pegida schon seit über 1 Jahr gesagt wird !!! 
Beispiel 7: Statusmeldung auf facebook.com/hasnain.kazim (2019) IN MEINEM HAUS BESTIMME IMMER NOCH ICH, WAS AUF DEN TISCH KOMMT!!!!! WEM'S NICHT PASST, KANN JA GEHEN!!!!!!

Beispiel 8: Statusmeldung auf facebook.com/spiegelonline (2018) ${ }^{7}$ Jetzt nehmen uns die Ausländer auch noch die Schönheit weg!!1!

Beispiel 6 zeigt Kommentare auf der Facebook-Präsenz der rechtspopulistischen Organisation Pegida. Sie können als Belege dafür gelten, dass die Registrierung des iterierten Ausrufezeichens als ,rechtspopulistisch' auf einer empirisch beschreibbaren Distribution des Zeichens in einschlägigen digitalen Diskursräumen beruht; dies müsste gesondert untersucht werden (vgl. auch Androutsopoulos 2019). Jedenfalls wird diese Distribution in der Registrierung des ,rechtspopulistischen' iterierten Ausrufezeichens präsupponiert (vgl. Spitzmüller 2013, S. 277). Wenn z.B. der Journalist Hasnain Kazim in einer Statusmeldung durch Versalienschreibung und mehrfachen Ausrufezeichen eine autoritäre Stimme stilisiert (Beispiel 7), ist dies nicht nur deshalb als Parodie erkennbar, dass diese grafischen Stilmittel bei Hasnain Kazim ansonsten nicht vorkommen, sondern auch deshalb, weil man unterstellt, dass ,Wutbürger“ eben mit Versalien und Iterationen schreiben. Gleiches gilt für die in Beispiel 8 gezeigte grafische Parodie, bei der ein oder mehrere Graphen des Ausrufezeichens durch die (wiederholte) Ziffer $<1>$ und gelegentlich ein ausgeschriebenes <elf> oder <eins > begleitet werden. Das Resultat - bei Androutsopoulos (2019) „Aufregezeichen“ genannt - wird im aktuellen deutschsprachigen Netzdiskurs als distanzierende Stilisierung einer nationalistischen bzw. rechtspopulistischen Position verstanden.

\section{Schlussfolgerungen und Ausblick}

Dieser Beitrag diskutiert Interpunktion als ein Teilsystem des Deutschen, an dem das Verhältnis zwischen Sprachwandel und dem übergreifenden soziokulturellen Wandelprozess der Digitalisierung besonders gut ablesbar ist. Interpunktionswandel wird an der Schnittstelle dreier Perspektiven untersucht: a) Distributive Umstrukturierung des Interpunktionsrepertoires im digitalen Sprachgebrauch,

7 Dies ist die journalistische Statusmeldung zu einem geteilten Medienbericht über rechtspopulistische Empörung in Österreich, ausgelöst durch die Wahl von Parsa Djawadiraad als „Mr. Kärnten“. 
aus der überaus frequente und nahezu obsolete Satzzeichen hervorgehen, b) Praktiken des Interpunktierens, die als Prozesse der Pragmatikalisierung, Syntaktisierung und konventionalisierten Ikonizität beschreibbar sind, sowie c) sprachideologische Verschiebungen, die zu einer Ent-Binarisierung in der Reflexion über digitale Interpunktion und zur sozialen Registrierung einzelner Interpunktionspraktiken führt. Diesen drei Analyseperspektiven ist gemeinsam, dass der gegenwärtig beobachtbare Interpunktionswandel nicht durch die eingesetzten Kommunikationstechnologien bedingt ist, sondern auf Bedarfe der visuellen Kontextualisierung unter Bedingungen schriftbasierter Interaktion reagiert. Die digitale Hard- und Software, die interaktionsorientiertes Schreiben erst ermöglicht, stecket ein Feld mit Spielräumen und Beschränkungen ab, in dem ein vorhandenes Zeicheninventar neue Gebrauchsmuster gewinnt, welche durch Konventionalisierung und öffentliche Sprachreflexion soziolinguistischen Wandel konsolidieren.

Der interaktionsorientierte Gebrauch von IPZ im informellen digitalen Schreiben geschieht nicht in einem sozialen Vakuum. Er setzt Traditionen privater Schriftlichkeit fort, die gesondert zu untersuchen wären ${ }^{8}$, und koexistiert mit der nach wie vor unangefochtenen orthografischen Normorientierung im institutionellen Schreiben. Welche Implikationen könnten die beschriebenen Interpunktionspraktiken für die standardsprachliche Zeichensetzung im Deutschen haben? Stellen sie - wie oft befürchtet - eine Bedrohung für das normorientierte Schreiben dar? Die Ergebnisse dieses Beitrags können diese Befürchtung nicht bestätigen, sondern zeigen gegenteilige Tendenzen auf. Ähnlich wie bei der Studie von Dürscheid/Wagner/Brommer (2010) liefern auch die Ergebnisse von Busch (2020) keine Belege für eine Auswirkung des informellen digitalen Schreibens auf die schulische Schriftlichkeit. Im Gegenteil wird deutlich, dass Schüler/innen sehr wohl zwischen dem je nach Handlungsbereich angemessenen Schreib- und damit auch Interpunktionsstil zu unterscheiden wissen. Festzuhalten ist abschließend: Die Digitalisierung des kommunikativen Alltags und die Verlagerung interpersonaler Kommunikation auf das Medium der digitalen Schriftlichkeit führen zu einer Ausfächerung des interaktionalen Umgangs mit Interpunktion, zur Herausbildung domänenspezifischer Schreib- bzw. Interpunktionsstile, zwischen denen gewechselt werden kann, und insgesamt zu einer schärferen Wahrnehmung kleinster grafischer Unterschiede, denen affektive und soziale Bedeutungen zugeschrieben werden.

8 Helga Kotthoff und Angelika Linke danke ich für Hinweise zu diesem Punkt. 


\section{Literatur}

Agha, Asif (2003): The social life of cultural value. In: Language and Communication 23, 3-4, S. 231-273.

Androutsopoulos, Jannis (2007): Neue Medien - neue Schriftlichkeit? In: Mitteilungen des Deutschen Germanistenverbandes 54, 1, S. 72-97.

Androutsopoulos, Jannis (2011): Language change and digital media: A review of conceptions and evidence. In: Kristiansen, Tore/Coupland, Nikolas (Hg.): Standard languages and language standards in a changing Europe. Oslo, S. 145-159.

Androutsopoulos, Jannis (Hg.) (2014): Mediatization and sociolinguistic change. (= linguae \& litterae 3). Berlin/Boston.

Androutsopoulos, Jannis (2017): Media and language change: Expanding the framework. In: Cotter, Colleen/Perrin, Daniel (Hg.): The Routledge handbook of language and media. London/New York, S. 403-423.

Androutsopoulos, Jannis (2018): Digitale Interpunktion: Stilistische Ressourcen und soziolinguistischer Wandel in der informellen digitalen Schriftlichkeit von Jugendlichen. In: Ziegler, Arne (Hg.): Jugendsprachen/Youth languages. Aktuelle Perspektiven internationaler Forschung/Current perspectives of international research. 2. Teilbd. Berlin/Boston, S. 721-748.

Androutsopoulos, Jannis (2019) Widerstand durch Zeichensetzung: Das Aufregezeichen als Mittel politischer Positionierung. In: Reflexivität kommunikativer Praktiken. Tagungsvortrag. Hamburg, 29.-30.8.2019. Universität Hamburg.

Androutsopoulos, Jannis (2020): Auslassungspunkte in der schriftbasierten Interaktion. Sequenziell-topologische Analyse an Daten von griechischen Jugendlichen. In: Androutsopoulos/Busch (Hg.), S. 130-155.

Androutsopoulos, Jannis/Busch, Florian (2020): Register des Graphischen: Skizze eines Forschungsansatzes. In: Androutsopoulos/Busch (Hg.), S. 1-29.

Androutsopoulos, Jannis/Busch, Florian (Hg.) (2020): Register des Graphischen. Variation, Interaktion und Reflexion in der digitalen Schriftlichkeit. (= Linguistik - Impulse \& Tendenzen 87). Berlin/Boston.

Auer, Peter (1986): Kontextualisierung. In: Studium Linguistik 19, S. 22-47.

Auer, Peter (1989): Natürlichkeit und Stil. In: Hinnenkamp, Volker/Selting, Margret (Hg.): Stil und Stilisierung. Tübingen, S. 27-59.

Baron, Naomi S. (2008): Always on: Language in an online and mobile world. Oxford/New York.

Baron, Naomi S./Ling, Richard (2011): Necessary smileys \& useless periods: Redefining punctuation in electronically-mediated communication. In: Visible Language 45, 1-2, S. 45-67.

Beißwenger, Michael/Storrer, Angelika (2012): Interaktionsorientiertes Schreiben und interaktive Lesespiele in der Chat-Kommunikation. In: Zeitschrift für Literaturwissenschaft und Linguistik 42, 4, S. 92-124.

Bieswanger, Markus (2013): Micro-linguistic structural features of computer-mediated communication. In: Herring, Susan/Stein, Dieter/Virtanen, Tuija (Hg.): Pragmatics of computer-mediated communication. (= Handbooks of Pragmatics 9). Berlin/Boston, S. 463-486.

Bilefsky, Dan (2016): Period. Full stop. Point. Whatever it's called, it's going out of style. In: The New York Times, 9.6.2016. Internet: http:/www.nytimes.com/2016/06/10/world/europe/ period-full-stop-point-whatever-its-called-millennials-arent-using-it.html (Stand: 25.7.2019). 
Bredel, Ursula (2011): Interpunktion. Heidelberg.

Britain, David (2016): Sedentarism and nomadism in the sociolinguistics of dialect. In: Coupland, Nikolas (Hg.): Sociolinguistics: Theoretical debates. Cambridge, S. 217-241.

Brommer, Sarah (2007): „Ein unglaubliches Schriftbild, von Rechtschreibung und Interpunktion ganz zu schweigen“. Die Schreibkompetenz der Jugendlichen im öffentlichen Diskurs. In: Zeitschrift für germanistische Linguistik 35, 3, S. 315-345.

Busch, Brigitta (2019): Sprachreflexion und Diskurs: Theorien und Methoden der Sprachideologieforschung. In: Antos, Gerd/Niehr, Thomas/Spitzmüller, Jürgen (Hg.): Handbuch Sprache im Urteil der Öffentlichkeit. (= Handbücher Sprachwissen 10). Berlin/Boston, S. 107-139.

Busch, Florian (2017): Informelle Interpunktion? Zeichensetzung im digitalen Schreiben von Jugendlichen. In: Der Deutschunterricht 69, 4, S. 87-91.

Busch, Florian (2020): Digitale Schreibregister. Kontexte, Formen und metapragmatische Reflexionen. Diss. Univ. Hamburg.

Coupland, Nikolas (2009): Dialects, standards and social change. In: Maegaard, Marie (Hg.): Language attitudes, standardization and language change. Perspectives on themes raised by Tore Kristiansen on the occasion of his 60th birthday. Oslo, S. 27-48.

Coupland, Nikolas (2014): Sociolinguistic change, vernacularization and broadcast British media. In: Androutsopoulos (Hg.), S. 67-96.

Crystal, David (2011): Internet Linguistics. A student guide. London.

Darics, Erika (2013): Non-verbal signalling in digital discourse. The case of letter repetition. In: Discourse, Context and Media 2, 3, S. 141-148.

Dürscheid, Christa/Frick, Karina (2016): Schreiben digital. Wie das Internet unsere Altagskommunikation verändert. (= Einsichten 3$)$. Stuttgart.

Dürscheid, Christa/Siever, Christina Margrit (2017): Jenseits des Alphabets - Kommunikation mit Emojis. In: Zeitschrift für germanistische Linguistik 45, 2, S. 256-285.

Dürscheid, Christa/Wagner, Franc/Brommer, Sarah (2010): Wie Jugendliche schreiben: Schreibkompetenz und neue Medien. (= Linguistik - Impulse \& Tendenzen 41). Berlin/New York.

Eckert, Penelope (2008): Variation and the indexical field. In: Journal of Sociolinguistics 12, 4, S. 453-476.

Gumperz, John J. (1992): Contextualization revisited. In: Auer, Peter/Di Luzio, Aldo (Hg.): The contextualization of language. (= Pragmatics and Beyond New Series 22). Amsterdam, S. 39-53.

Gunraj, Danielle N./Drumm-Hewitt, April M./Dashow, Erica M./Upadhyay, Sri Siddhi N./Klin, Celia M. (2016): Texting insincerely: The role of the period in text messaging. In: Computers in Human Behavior 55, B, S. 1067-1075.

Günthner, Susanne/Mutz, Katrin (2004): Grammaticalization vs. pragmaticalization? The development of pragmatic markers in German and Italian. In: Bisang, Walter/Himmelmann, Nikolaus P./Wiemer, Björn (Hg.): What makes Grammaticalization? A look from its fringes and its components. (= Trends in Linguistics. Studies and Monographs 158). Berlin/Boston, S. 77-107.

Haase, Martin/Huber, Michael/Krumeich, Alexander/Rehm, Georg (1997): Internetkommunikation und Sprachwandel. In: Weingarten, Rüdiger (Hg.): Sprachwandel durch Computer. Opladen, S. 51-85.

Herring, Susan C./Zelenkauskaite, Asta (2008): Gendered typography: Abbreviation and insertion in Italian iTV SMS. In: Siegel, Jason F./Nagle, Traci C./Lorente-Lapole, Amandine/Auger, Julie. (Hg.): IUWPL7: Gender in language, classic questions, new contexts. Bloomington, S. 73-92.

Houghton, Kenneth J./Upadhyay, Sri Siddhi N./Klin, Celia M. (2018): Punctuation in text messages may convey abruptness. Period. In: Computers in Human Behavior 80, S. 112-121. 
JIM-Studie (2018): Jugend, Information, Medien. Basisuntersuchung zum Medienumgang 12- bis 19-Jähriger. Stuttgart.

Jones, Rodney H./Chik, Alice/Hafner, Christoph A. (Hg.) (2015): Discourse and digital practices. Doing discourse analysis in the digital age. London.

Jucker, Andreas/Dürscheid, Christa (2012): The linguistics of keyboard-to-screen communication: A new terminological framework. In: Linguistik Online 56, S. 39-64. Internet: https:// doi. org/10.13092/lo.56.255 (Stand: 25.7.2019).

Kemper, Anna (2016): !!!!!!!!!!!!!!!!!!!!!!!!!!!!!!. In: Zeit Magazin, 6.1.2016. Internet: https:// www.zeit.de/zeit-magazin/leben/2016-01/til-schweiger-tatort-ausrufezeichen (Stand: 25.7.2019).

Krotz, Friedrich/Despotovic, Cathrin/Kruse, Merle-Marie (Hg.) (2017): Mediatisierung als Metaprozess. Transformationen, Formen der Entwicklung und die Generierung von Neuem. Wiesbaden.

McSweeney, Michelle (2018): The pragmatics of text messaging. Making meaning in messages. New York/London.

Meibauer, Jörg (2007): Syngrapheme als pragmatische Indikatoren: Anführung und Auslassung. In: Döring, Sandra/Geilfuß-Wolfgang, Jochen (Hg.): Von der Pragmatik zur Grammatik. Leipzig, S. 21-37.

Ong, Kenneth Keng Wee (2011): Disagreement, confusion, disapproval, turn elicitation and floor holding. Actions as accomplished by ellipsis marks-only turns and blank turns in quasisynchronous chats. In: Discourse Studies 13, 2, S. 211-234.

Pappert, Steffen (2017): Zu kommunikativen Funktionen von Emojis in der WhatsAppKommunikation. In: Beißwenger, Michael (Hg.): Empirische Erforschung internetbasierter Kommunikation. (= Empirical Linguistics 9). Berlin/Boston, S. 175-212.

Raclaw, Joshua (2006): Punctuation as social action: The ellipsis as a discourse marker in computer-mediated communication. In: Proceedings of the 32nd annual meeting of the Berkeley Linguistic Society. Berkeley, S. 299-306. Internet: http://dx.doi.org/10.3765/bls. v32i1.3469 (Stand: 25.7.2019).

Sanchez-Stockhammer, Christina (2016): Punctuation as an indication of register: Comics and academic texts. In: Schubert, Christoph/Sanchez-Stockhammer, Christina (Hg.): Variational text linguistics. Revisiting register in English. (= Topics in English Linguistics 90). Berlin/ Boston, S. 139-168.

Sandig, Barbara (2006): Textstilistik des Deutschen. 2., völlig neu bearb. u. erw. Aufl. Berlin/ Boston.

Spitzmüller, Jürgen (2013): Metapragmatik, Indexikalität, soziale Registrierung. Zur diskursiven Konstruktion sprachideologischer Positionen. In: Zeitschrift für Diskursforschung 3, S. 263-287.

Squires, Lauren (2010): Enregistering internet language. In: Language in Society 39, 4, S. 457-492.

Squires, Lauren (2012): Whos punctuating what? Sociolinguistic variation in instant messaging. In: Jaffe, Alexandra/Androutsopoulos, Jannis/Sebba, Mark/Johnson, Sally (Hg.): Orthography as social action. Scripts, spelling, identity and power. (= Language and Social Processes 3 ). Berlin/Boston, S. 289-324.

Tagliamonte, Sali A. (2014): Situating media influence in sociolinguistic context. In: Journal of Sociolinguistics 18, 2, S. 223-232.

Tannen, Deborah (2013): The medium is the metamessage: Conversational style in new media interaction. In: Tannen, Deborah/Trester, Anna Marie (Hg.): Discourse 2.0: Language and new media. Washington, S. 99-117.

Uhlenberg, Marlene (2016): Interpunktion in digital vermittelter Interaktion. MA. Univ. Hamburg. 
Vandergriff, Ilona (2013): Emotive communication online: A contextual analysis of computermediated communication (CMC) cues. In: Journal of Pragmatics 51, S. 1-12.

Wichter, Sigurd (1991): Zur Computerwortschatz-Ausbreitung in die Gemeinsprache: Elemente der vertikalen Sprachgeschichte einer Sache. (= Germanistische Arbeiten zu Sprache und Kulturgeschichte 17). Frankfurt a.M. u.a. 